\title{
DE PRONASOL A OPORTUNIDADES: POLÍTICA SOCIAL Y PERSISTENCIA DE LA POBREZA EN MÉXICO
}

\author{
FROM PRONASOL TO OPORTUNIDADES: SOCIAL POLICY AND \\ ENDURING POVERTY IN MEXICO
}

\author{
Félix Acosta Díaz \\ Colegio de la Frontera Norte, México/Mexico \\ acosta@colef.mx
}

Recibido/Received: 11/06/2010

Aceptado/Accepted: 30/07/2010

\section{RESUMEN}

Después de casi tres lustros de política social, la pobreza extrema parece haberse convertido ya en un problema persistente en México. Aún en los años en que las condiciones económicas han sido favorables, la población mexicana sufre los efectos de la pobreza y la carencia de oportunidades sociales. El problema se agrava ante las contingencias económicas. El propósito general de este trabajo es doble: por una parte, contrastar los enunciados y los resultados de la política social de atención a la pobreza en México en el período posterior a la gran crisis de 1982; por otra parte, señalar las expectativas y las posibilidades de una nueva política social que permita lograr un avance más eficaz de las decisiones y las acciones de política pública orientadas a combatir la pobreza y la exclusión social en el México moderno.

\section{PALABRAS CLAVE}

Exclusión social, gobernanza, políticas públicas, administración pública, actores sociales, ley general de desarrollo social.

\section{SUMARIO}

1. Introducción. 2. Crisis económica y crisis del estado del bienestar. 3. La política social en la etapa del auge neoliberal: de PRONASOL a OPORTUNIDADES. 4. ¿Hacia una nueva política social?. 5. Los resultados de la política social. 6. Consideraciones finales. Bibliografia.

\footnotetext{
ABSTRACT

After almost fifteen years of social policies, extreme poverty has become an enduring problem in Mexico. Even in those years when nation-wide economic conditions have been particularly good, an important segment of the Mexican population faces the problem of poverty and the lack of social opportunities and this segment grows heavily with economic turndowns. The purpose of this paper is twofold: on one hand, it compares the objectives against the efficacy of social policy aimed to alleviate and reduce poverty in Mexico in the period after the great 1982 economic and social crisis; on the other hand, it points out the expectations and the problems of a new social policy that may be more effective in overcoming poverty and social deprivation in modern Mexico.
} 


\section{KEYWORDS}

Social deprivation, governance, public policy, public administration, social actors, general law of social development.

\section{CONTENTS}

1. Introduction. 2. Economic crisis and the welfare state crisis. 3. Social policy during the neoliberal stage: from PRONASOL to OPORTUNIDADES. 4. Towards a new social policy? 5. The efficacy of social policy. 6. Final remarks. References.

\section{INTRODUCCIÓN}

El propósito general de este trabajo es doble: por una parte, contrastar los enunciados y los resultados de la política social de atención a la pobreza en México en el período posterior a la gran crisis de 1982; por otra parte, señalar las expectativas y las posibilidades de una nueva política social que permita lograr un avance más eficaz de las decisiones y las acciones de política pública orientadas a combatir la pobreza y la exclusión social en el México moderno.

Para ello, en la segunda sección se presentan los antecedentes económicos y sociales que culminan con la desaparición del estado del bienestar; en la tercera sección se presentan las principales características de los programas de atención a la pobreza en la etapa del auge neoliberal; en la cuarta sección se revisan las posibilidades de contar con una política social que incorpore efectivamente la participación de los diferentes actores públicos y sociales y los organice en la responsabilidad de erradicar la pobreza y la exclusión social; en la quinta sección se presentan algunos resultados que permiten evaluar la efectividad de la política social en el período reciente; finalmente, se ofrecen algunas recomendaciones para darle mayor viabilidad a la política social.

\section{CRISIS ECONÓMICA Y CRISIS DEL ESTADO DEL BIENESTAR}

Los orígenes y características de la política social en la etapa moderna de México se pueden trazar en los acontecimientos económicos y sociales que ocurren en la segunda mitad del siglo XX. El contexto económico y social que caracteriza a México en ese período puede ser dividido en dos partes muy disímbolas en términos de sus características económicas y sociales: la primera, que tiene sus orígenes en los años inmediatamente posteriores a la segunda guerra mundial, está caracterizada por el crecimiento económico y el auge del estado del bienestar, particularmente importantes a partir de la década de los años cuarenta; a la segunda parte, que se hace especialmente visible a partir de 1982, la distingue precisamente lo contrario, es decir, la recesión económica, los programas gubernamentales de ajuste fiscal y restructuración económica, y el abandono del estado del bienestar como política de atención universalista a las necesidades de la población (Hernández Laos, 1999; Reygadas, 2008; ver también Aguilar, 2006, 2010). 
En este trabajo tenemos especial interés en el segundo de estos periodos, pues consideramos que la gran crisis económica y social de 1982 y los obligados ajustes fiscales del gobierno modificaron sustantivamente el papel del estado en la conducción de los procesos sociales y el enfoque de atención a problemas como la desigualdad y la pobreza, pasando de las políticas universales que no hacían distinciones en términos de las características de la población, a las políticas sociales asistenciales, focalizadas y centradas en el mejoramiento de las capacidades individuales.

Sin embargo, consideramos que para entender mejor la magnitud de los cambios políticos y sociales que ocurren en México con la gran crisis económica de 1982 es necesario retroceder un poco en el tiempo y considerar con un poco más de detenimiento lo que sucedió con la economía mexicana y las variables sociales en los años de 1978 a 1981.

En 1976, en el contexto de la recesión económica de 1973-1975 de los países industrializados, se vuelve evidente en México y en el resto de los países de América Latina el agotamiento de un modelo de crecimiento apoyado en el sector industrial principalmente la manufactura- y basado en la sustitución de importaciones, que en México estuvo orientada principalmente al reemplazo de las importaciones de bienes de consumo y en menor medida de los bienes intermedios.

Entre los problemas que se hacen evidentes en ese año destacan la insuficiencia de la economía en la creación de empleos, la escasa articulación del aparato industrial, la fuerte dependencia del exterior en el abastecimiento de bienes de capital, la falta de competitividad internacional de los productos mexicanos, el fuerte déficit financiero del gobierno, el endeudamiento externo y, finalmente, un proceso inflacionario de desconocida intensidad para la experiencia mexicana de la posguerra (CEPAL, 1977; Marini, 1984).

Los fuertes desequilibrios observados en la balanza de pagos y la acumulación de alzas de precios exageradas en relación a las de los países con los que se comerciaba se conjugaron con otros factores para que el gobierno decidiera abandonar el régimen histórico del tipo de cambio controlado y como resultado, el tipo de cambio, que había permanecido fijo en 12.49 pesos por dólar desde 1954 y que representaba un símbolo de la estabilidad económica y financiera del país, se incrementó a cerca de 20.00 pesos en septiembre de 1976 (CEPAL, 1977).

El programa de ajuste que el gobierno instrumentó estuvo dirigido principalmente a resolver los desequilibrios financieros internacionales en las cuentas internas, además de contener el proceso inflacionario. Para hacer posible este último objetivo, el gobierno adoptó una política de contención salarial que se ha mantenido vigente y que ha provocado desde entonces importantes disminuciones en los ingresos reales y en el bienestar de la población (CEPAL, 1978, 1984).

Sin embargo, el descubrimiento en 1978 de cuantiosas reservas de petróleo modificó sustancialmente las perspectivas de México y le concedió al gobierno de José López Portillo (1976-1982) un margen más amplio para llevar a cabo su programa de ajuste y planear la recuperación económica del país. Así, basado en la expansión de la producción petrolera, México pudo, con mayor éxito que el resto de los países de América Latina, sortear los efectos de la recesión económica y alcanzar inclusive tasas de crecimiento históricas -por arriba del 8\% anual- en el período 1978-1981.

En esos tres años, las necesidades crecientes de inversión pública y privada superaron con creces la capacidad de ahorro interno y la capacidad de captación del sistema bancario. Entonces, apoyados por las perspectivas de crecimiento, el gobierno y 
las empresas privadas recurrieron cada vez en mayor medida al financiamiento externo, aún en condiciones muy desventajosas. En consecuencia, la deuda pública externa aumentó de 22 mil a 53 mil millones de dólares de 1977 a 1981, mientras que la deuda de las empresas privadas lo hizo de 7 mil a 19 mil millones de dólares. Además, las condiciones de contratación de la deuda se volvieron particularmente desfavorables a finales de 1981 (CEPAL, 1984).

El breve "milagro mexicano" terminó en 1982. El aumento de las tasas de interés internacionales, la caída de los precios del petróleo, el agotamiento de las fuentes externas de financiamiento y la salida masiva de capitales mexicanos mostraron que la vulnerabilidad del sistema económico mexicano se había vuelto mayor con el auge petrolero. La serie de medidas -devaluaciones sucesivas, ajuste fiscal, contracción salarial, entre otras- adoptadas por el gobierno de Miguel de la Madrid (1982-1988) para contener la crisis en 1982 provocaron, entre otras cosas, que la economía se contrajera en $0.5 \%$, que la inflación aumentara a una tasa anual de $98.8 \%$ y que el salario mínimo, usado en esos años como referencia para ubicar a la población en pobreza, perdiera un $12.2 \%$ adicional de su poder adquisitivo (CEPAL, 1984, 1985).

Pero lo más difícil todavía no sucedía. En 1983, la crisis económica se manifestó ya como un proceso generalizado de recesión económica con elevada inflación que se extendió durante todo el gobierno de Miguel de la Madrid. La puesta en marcha del Programa Inmediato de Reordenación Económica, el cuál contó con el aval y apoyo del Fondo Monetario Internacional inauguró ya definitivamente para los mexicanos un largo y doloroso período de ajuste social cuyas consecuencias resultaron especialmente negativas para los sectores más pobres de la población, tanto en las áreas rurales como en las áreas urbanas.

Así, desde 1982 hasta 1988, los ajustes fiscal y financiero fueron la prioridad para el gobierno de Miguel de la Madrid. Sin embargo, el proceso de ajuste no siguió un proceso lineal y la vulnerabilidad del sistema económico ante los impactos externos se volvió a hacer evidente otra vez en 1986, año en el que no se contó con financiamiento externo y el gobierno dejó de recibir cerca de 8,500 millones de dólares por la caída en el precio del petróleo. Como resultado, el producto interno bruto cayó ese año en $3.6 \%$ y la inflación alcanzó un 105.7\% (CEPAL, 1988, 1990).

Lo que sí siguió un proceso lineal fue el deterioro de los salarios reales, con consecuencias especialmente negativas apara las familias de bajos ingresos y de los sectores medios. Con las disminuciones sucesivas de 1982 a 1988, el salario mínimo llegó a tener a finales del gobierno de Miguel de la Madrid un 47\% del poder adquisitivo que tenía en 1976 y la participación de los salarios en el producto interno bruto disminuyó de cerca de 40\% antes de la crisis de 1982 a un porcentaje apenas superior a 20\% en 1988 (CEPAL, 1989). Para analizar los efectos de la crisis económica sobre el bienestar de la población, Cortés, Hernández Laos y Rubalcava (1990) analizaron los salarios de cotización en el sistema de seguridad social y sus resultados apoyan adicionalmente la hipótesis de una creciente polarización del ingreso en México en el período de 1982 a 1986.

El proceso de ajuste continuó todavía durante el gobierno de Carlos Salinas (1988-1994); no obstante, las políticas neoliberales y neogerencialistas (ver Dunleavy y Hood, 1994; Hood, 1991; Osbourne y Gaebler, 1992; y Osbourne y Plastrik, 1999) de adelgazamiento del Estado mediante la venta de importantes empresas públicas como Teléfonos de México y la privatización de otros bienes y servicios tradicionalmente 
ofrecidos por el gobierno, junto con la estabilización de la inflación, permitieron que la economía mexicana reiniciara, aunque temporalmente, el camino del crecimiento. Así, entre 1988 y 1994, el producto interno bruto se mantuvo creciendo a tasas anuales promedio cercanas al 3\%, mientras que la inflación descendió de manera sistemática después de su máximo histórico de $159.2 \%$ en 1987 hasta un $7.1 \%$ en 1994, año en el que se volvió a hacer evidente la vulnerabilidad del sistema financiero mexicano y la fragilidad de la recuperación económica.

Sin embargo, el respiro que se logró en las finanzas del gobierno con los recursos obtenidos de la venta de empresas públicas y con la recuperación económica le permitió a la administración del presidente Carlos Salinas echar a andar el primer programa nacional de combate a la pobreza en México, para tratar de mitigar los efectos negativos acumulados de la recesión económica y el ajuste fiscal del gobierno sobre los sectores más desfavorecidos de la población. De esta manera, termina "oficialmente" el estado del bienestar en México y se inaugura una primera etapa de la política social en el México moderno, que incluye a un grupo de estrategias asistenciales y focalizadas de atención a los pobres que pasa de PRONASOL en el gobierno de Carlos Salinas (1988-1994), a PROGRESA en el gobierno de Ernesto Zedillo (1994-2000) y a OPORTUNIDADES en el gobierno de Vicente Fox (2000-2006).

\section{LA POLÍtica SOCIAL EN LA ETAPA DEL AUGE NEOLIBERAL: DE PRONASOL A OPORTUNIDADES}

El PRONASOL fue anunciado por el presidente Carlos Salinas el día de su toma de posesión como la columna vertebral de la política social de su gobierno, orientada a aliviar las consecuencias sociales del doloroso proceso de ajuste y restructuración económica iniciado desde el gobierno de Miguel de la Madrid y a aminorar la tensión política derivada del difícil proceso electoral. Concebido inicialmente con una racionalidad neogerencial aplicada en todos los ámbitos de la administración pública -que descansaba en el principio de la eficiencia en el uso de los recursos públicos- el programa hacía énfasis en la participación social comunitaria y estableció como poblaciones prioritarias de atención a los indígenas, a los habitantes de las zonas áridas y a los pobres extremos rurales y urbanos.

Sin embargo, debido a la débil legitimidad política de su gobierno, ocasionada por la falta de credibilidad social en los resultados de las elecciones de 1988, la naturaleza y las características operativas del programa fueron diseñadas también para construir una amplia base social y política que le permitiera al gobierno construir esa legitimidad y restarle espacios al avance de la izquierda mexicana, representada por el Partido de la Revolución Democrática, el principal opositor del gobierno, el cual se organizó y constituyó inmediatamente después del proceso electoral como una coalición de los principales partidos de izquierda (Díaz-Cayeros y Magaloni, 2003).

Por estas razones, aunque la participación social a nivel comunitario era el elemento central en la discusión, la toma de decisiones y la implementación de los proyectos financiados por el programa, el PRONASOL era administrado con una lógica burocrática y centralizada al más puro estilo weberiano (Weber, 1996). El segundo día de su mandato, el presidente Salinas anunció la creación de una Comisión (ver Consejo 
Consultivo del Programa Nacional de Solidaridad, 1994) encargada del programa encabezada por él mismo e integrada por los titulares de las diferentes dependencias del gobierno federal relacionadas con las tareas del bienestar social y nombró a un Coordinador del programa. Eventualmente, esa Coordinación pasó a formar parte de la Secretaría de Desarrollo Social (SEDESOL) cuando ésta se creó en 1992 y desde entonces se mantiene en esa dependencia la coordinación del mayor programa de atención a los pobres. Con ésta lógica burocrática y centralizada, la administración del programa era nacional y la operación recaía en delegados estatales nombrados por el titular de la SEDESOL y esa estructura de administración de los programas del gobierno federal orientados a combatir la pobreza se mantiene hasta la actualidad.

Sin embargo, aunque los recursos del PRONASOL eran administrados centralmente, la gestión del programa descansaba ampliamente en la participación social comunitaria, organizada en los llamados Comités de Solidaridad, generando una representación directa de las diferentes comunidades rurales y urbanas en la gestión y solución de los diferentes problemas a nivel de comunidad.

Quizá por esa necesidad de ampliar la base social del gobierno del presidente Carlos Salinas, el programa no siguió estrictamente una estrategia focalizada en su operación y los Comités de Solidaridad se extendieron por todo el país, generando una estrecha relación clientelar entre el gobierno y los beneficiarios del programa. Esta relación clientelar le permitió al Partido Revolucionario Institucional (PRI), volver a ganar las elecciones presidenciales en 1994, a pesar de una extendida crisis política ocasionada por los asesinatos de Luis Donaldo Colosio -primer candidato del PRI en esas eleccionesde Francisco Ruiz Massieu -presidente del mismo partido- y de un cardenal de la iglesia católica.

La crisis financiera de 1994, que culminó con el rescate bancario por parte de la administración de Ernesto Zedillo -con el apoyo del gobierno de los Estados Unidos- y con la conversión de los pasivos de los bancos en deuda pública interna mediante el programa FOBAPROA, provocó que el gobierno pusiera mayor énfasis en la eficiencia económica en la operación de los programas sociales. Después de algunos años de parálisis, la política social del gobierno de Ernesto Zedillo sustituyó el PRONASOL del gobierno de Carlos Salinas por el programa PROGRESA en 1997 y la eficiencia económica mediante la focalización se convirtió en el principio rector de las acciones del gobierno orientadas a mitigar los efectos de este nuevo ajuste sobre los viejos y los nuevos pobres del país, especialmente los pobres de las zonas rurales, hacia quienes se orientaron las acciones del programa. PROGRESA fue renombrado como OPORTUNIDADES en el segundo año del gobierno de Vicente Fox, pero mantuvo sus características asistencialistas y focalizadas, aunque ante el crecimiento reciente de la pobreza, ha tenido que incluir a los pobres urbanos dentro de la población atendida.

La racionalidad neogerencial de ambos programas de atención a la pobreza descansa en un trabajo del influyente economista mexicano Santiago Levy, preparado durante su estancia en el Banco Mundial. La propuesta de Levy (1991) parte de la premisa de que el gasto del gobierno en los pobres extremos debe ser visto como una inversión que, si se mantiene de manera sistemática, permitirá que los pobres incrementen su potencial productivo y contribuyan al aumento del ingreso nacional. 
Para decidir cuántos recursos necesitaba el gobierno invertir en los pobres extremos, Levy realizó un diagnóstico de la pobreza estableciendo una línea monetaria de pobreza extrema basada en estándares de ingesta de calorías bajo el supuesto de que la desnutrición constituye un reflejo de la falta de recursos monetarios en las familias para adquirir cantidades adecuadas de alimentos. De acuerdo a sus cálculos con datos de la Encuesta Nacional de Ingresos y Gastos de los Hogares (ENIGH) de 1984, mientras que la pobreza extrema era un problema mayormente rural (los niveles de incidencia de la pobreza que obtuvo fueron de $19.5 \%$ a nivel nacional, $37.2 \%$ en las áreas rurales, y $9.9 \%$ en las áreas urbanas), la mayor parte de los beneficios entregados por el PRONASOL través de subsidios a alimentos básicos como la tortilla y la leche se entregaban a los pobres moderados de las zonas urbanas y metropolitanas como el Distrito Federal.

En su diagnóstico sobre la pobreza en México, Levy postula que las raíces de la pobreza se encuentran en las áreas rurales y en el sesgo urbano de las políticas de desarrollo impulsadas por el gobierno a partir de la década de los sesenta y que el exceso de regulación en el sector agrícola, -especialmente en lo que se refiere a la persistencia de formas comunales y familiares de tenencia de la tierra heredadas desde la reforma agraria impulsada por el presidente Lázaro Cárdenas- han impedido que la inversión privada impulse el crecimiento económico de ese sector y que la fuerza de trabajo agrícola se incorpore a los mercados de trabajo remunerados.

Por lo tanto, para erradicar la pobreza Levy sostiene que se requieren dos tipos de políticas públicas: unas dedicadas a crear condiciones para que los pobres rurales extremos se incorporen "productivamente" a los mercados laborales de trabajo; otras, orientadas a combatir las causas institucionales de la pobreza, debían enfocarse a superar los sesgos urbanos en la inversión pública en infraestructura, salud y educación y a modificar las condiciones -con énfasis en las que se refieren a las formas comunales de tenencia de la tierra- que impiden que el campo se beneficie de la inversión privada.

En relación al diseño de la política social de atención a la pobreza, para Levy era fundamental tomar en cuenta que en los pobres extremos rurales persisten características, necesidades y comportamientos que crean condiciones de complementariedad para que las familias rurales queden atrapadas en un "círculo vicioso" de reproducción de la pobreza. Estas complementariedades se refieren en primer lugar a sus altos niveles de fecundidad, los cuales están asociados a su vez a altas tasas de mortalidad infantil, a las carencias en educación y a la falta de acceso a los métodos anticonceptivos; en este contexto, tener muchos hijos puede ser visto como un comportamiento racional por parte de las familias rurales pobres.

En segundo lugar, los pobres extremos rurales no pueden reaccionar ante una disminución transitoria en los salarios reales trabajando un mayor número de horas, debido a que ya dedican todo su tiempo al trabajo en el campo y a que enfrentan restricciones de movilidad hacia los mercados de trabajo; por estas razones, las crisis económicas tienen un efecto inmediato sobre sus ingresos y sus condiciones nutricionales.

En tercer lugar, los hijos de los pobres rurales no pueden beneficiarse de la ampliación de las oportunidades de educación creadas por el gobierno porque tienden a incorporarse a edades tempranas a tareas familiares o al trabajo en la parcela familiar y a que sus padres no tienen tampoco incentivos para mandarlos a la escuela porque hacerlo implicaría dejar de contar con su fuerza de trabajo. 
En cuarto lugar, debido a que los pobres extremos rurales no poseen activos ni tiene acceso al crédito, el deterioro en las condiciones económicas generales ante una crisis económica se traduce inmediatamente en menores niveles de consumo y mayores niveles de desnutrición.

En quinto lugar, debido a su condición de pobreza, las familias rurales mantienen una dieta compuesta mayormente por calorías y su demanda de alimentos con mejores contenidos de nutrientes es prácticamente nula, aún en condiciones de relativa mejoría en los ingresos. En sexto lugar, estos hábitos alimenticios tienen un efecto negativo directo en la productividad de los pobres extremos rurales. Y finalmente, en los comportamientos internos de las familias rurales pobres persisten inequidades internas que afectan mayormente a los niños.

Como se ha mencionado en renglones anteriores, este diagnóstico acerca de las características, las necesidades y el comportamiento de los pobres extremos rurales constituye el fundamento y la racionalidad asistencial y focalizada en el diseño e implementación de los programas PROGRESA y OPORTUNIDADES (Coady, 2003; Orozco y Hubert, 2005), los cuales han constituido la base de la política social del gobierno mexicano desde 1997.

Desde ese año, el gobierno federal entrega directamente a través de estos programas a las madres de las familias en condiciones de pobreza un paquete de beneficios económicos marginales -para no crear distorsiones en el sistema de precios y en los mercados de bienes y de trabajo- que se encuentran condicionados a que los hijos de estas familias asistan regularmente a la escuela y a que sus madres y ellos acudan periódicamente a clínicas médicas de atención preventiva en materia de fecundidad, uso de anticonceptivos, salud materna e infantil y nutrición, mientras que en la economía se crean las condiciones adecuadas para que los pobres rurales extremos puedan convertir esas nuevas capacidades y comportamientos en oportunidades de desarrollo individual y familiar.

Sin embargo, ante la persistencia de la pobreza y su expansión reciente hacia las áreas urbanas, la población atendida por estos programas ha crecido de 2.6 millones de familias con PROGRESA en 1997 a casi seis millones de familias con OPORTUNIDADES en 2010.

\section{4. ¿HACIA UNA NUEVA POLÍTICA SOCIAL?}

La aprobación de La Ley General de Desarrollo Social -en adelante LGDS(DOF, 2004) en enero de 2004 constituye un cambio significativo en la política social en el México moderno por dos razones que remiten tanto a su concepción analítica como a sus características como un sistema de acción pública deliberadamente orientado a resolver problemas sociales como la pobreza y la exclusión social (Aguilar, 2008). En primer lugar, el hecho de que la política social haya sido elevada al rango de precepto legal constituye un paso importante para convertirla en una política social de estado, cuyos objetivos, principios y funcionamiento trasciendan las diferentes administraciones sexenales del gobierno federal, dándole mayor certidumbre a todos los actores públicos y sociales involucrados en los diferentes aspectos de la política. 
En segundo lugar, el hecho de que la LGDS haya sido primero impulsada por los partidos de oposición al partido del gobierno, pero finalmente aprobada por todos los partidos políticos representados en el poder legislativo nos anima a establecer en un principio la premisa de que la política social concebida en ella puede ser conceptualizada y analizada como un conjunto de creencias valorativas compartidas producto de algún mecanismo de diálogo y deliberación aceptado e implementado por los diferentes actores públicos y sociales respecto a problemas sociales no deseados para la población.

Desde nuestro punto de vista, la LGDS (en adelante Ley) concibe en términos generales e intencionales a la política social como una propuesta o un sistema de acción de gobernar en modo de cogobernanza por tres razones distintivas que remiten a las referencias teóricas y conceptuales de la nueva gobernanza y de la sociología del conocimiento (ver Aguilar, 2008a, 2008b; Kooiman, 2004 y Kooiman et al, 2008; ver también, Berger y Luckman, 1967; Giddens, 1984; y Pzeworski, 1982). En primer lugar, la Ley puede ser concebida como el acuerdo o conjunto de acuerdos de los diferentes actores sociales involucrados en la discusión y solución de los problemas que son objeto de la Ley y que la acción del gobierno convierte en problemas públicos cuya solución descansa en varios principios aceptados por los diferentes actores.

En segundo lugar, la Ley crea un Sistema Nacional de Desarrollo Social (SNDS) el cual es concebido como un mecanismo de constante concurrencia en el que están señaladas las obligaciones y las responsabilidades de los diferentes actores públicos y sociales en el diseño, la implementación, el monitoreo y la evaluación de la política social. El SNDS está constituido por un conjunto de instituciones con atribuciones y responsabilidades específicas en las tareas de formulación, implementación, coordinación y evaluación de la política social: la Comisión Nacional de Desarrollo Social (CNDS); la Comisión Intersecretarial de Desarrollo Social (CIDS); el Consejo Consultivo de Desarrollo Social (CCDS); y el Consejo Nacional de Evaluación de la Política de Desarrollo Social (CONEVAL). Finalmente, al menos en los enunciados principales de la Ley, la participación social y la rendición social de cuentas parecen constituir elementos distintivos de la nueva política social.

Sin embargo, el hecho de que la política social haya sido elevada al rango de precepto legal y que su concepción tenga algunos de los elementos distintivos de la cogobernanza no garantiza, como lo establece el marco teórico-conceptual de referencia, que todos los objetivos y los principios de la LGDS se cumplan en la práctica y que en las instituciones encargadas de su diseño, implementación y evaluación existan espacios que garanticen o al menos favorezcan la cogobernanza como proceso de gobierno. Dos elementos que distinguen al proceso de gobernar en modo de cogobernanza son el necesario acompañamiento del estado y el gobierno por parte de los diversos actores de la sociedad y la eficacia social de las acciones del gobierno en la solución de problemas sociales y en la creación de oportunidades (Aguilar, 2008a; Kooiman, 2004; Kooiman et al, 2008). Por su parte, Aguilar (2008a) ha señalado también que la indagación de la calidad institucional de una propuesta de cogobernanza está íntimamente relacionada con la densidad y la calidad de la participación social.

Señalamos en seguida algunos problemas o inconsistencias de la política social que pueden estar limitando sus posibilidades como propuesta de cogobernanza: el primero se refiere a las limitaciones de la participación social en las instituciones que conforman el 
SNDS; el segundo problema está relacionado con la factibilidad administrativa de la política social en el contexto actual de la administración pública federal mexicana.

Un primer problema se refiere al propio marco legal que sustenta a la política social y tiene que ver con la densidad y la calidad de la participación social en la política social. Aunque en la LGDS se reconoce el lugar que debe ocupar la participación social en la política social, ésta tiene densidades y cualidades diferentes en cada una de las instituciones que conforman el SNDS y en algunas es prácticamente inexistente.

En primer lugar está la CNDS: en esta comisión, que tiene como propósito la consideración de la integralidad de los esfuerzos de los diferentes niveles de gobierno y el papel que juegan las inequidades regionales, no participan ni actores de la iniciativa privada ni del resto de la sociedad. En segundo lugar está la CIDS: en esta comisión, que tiene la responsabilidad de asegurar la coordinación de las acciones y programas del gobierno federal en la política social no participan tampoco representantes del sector privado -asociaciones, confederaciones o agrupaciones empresariales- que tienen un peso fundamental en la generación de oportunidades de empleo, ingreso, salud y seguridad social ni de la sociedad civil organizada en torno a demandas específicas de grupos minoritarios o vulnerables. En tercer lugar está el CCDS: éste es un consejo consultivo que tiene atribuciones para emitir recomendaciones para mejorar la política social, promover la colaboración de organismos públicos y privados, nacionales e internacionales e impulsar "la participación ciudadana y de las organizaciones" en el seguimiento, operación y evaluación de la política social, y está constituido por ciudadanos de los sectores privado y social y de los ámbitos académico, profesional, científico y cultural. Finalmente está el CONEVAL, institución del gobierno responsable de medir la pobreza y evaluar la política social, integrado por dos representantes del gobierno y seis académicos especialistas en evaluación de la política social, quienes constituyen el soporte técnicocientífico de las tareas de evaluación y medición de la pobreza del consejo; aquí también está ausente la participación de representantes del sector privado o de la sociedad organizada en torno a necesidades o intereses específicos de grupos sociales.

Un segundo problema que puede estar afectando la viabilidad de la política social está relacionado con las tensiones entre los preceptos y las instituciones de la política social y el funcionamiento actual de la estructura administrativa del gobierno, el cual conserva muchas de sus tradiciones jerárquicas y burocráticas. En primer lugar, en un sistema presidencialista como el de México, que tiene raíces profundas en el modo jerárquico de gobernar, pareciera que ninguna decisión de gobierno es válida y cumplida por los titulares de las diferentes dependencias federales si en la decisión no participa el propio jefe del ejecutivo federal y este modo jerárquico y burocrático de gobernar la política pública a nivel federal se reproduce en los niveles estatales y municipales del gobierno e influye en la calidad de las interacciones entre los actores públicos y entre éstos y los actores sociales.

En esta lógica, los trabajos de la CIDS pueden estar condenados al fracaso porque es difícil que los titulares del resto de las dependencias federales atiendan los acuerdos de una comisión que es presidida por un par y no por el presidente, y porque los asuntos y los acuerdos verdaderos y válidos sobre la materia se tomen en el seno del gabinete social, en el que sí participa el jefe del ejecutivo federal estableciendo 
compromisos y esquemas de rendición de cuentas para los integrantes de su gabinete de gobierno.

\section{LOS RESULTADOS DE LA POLÍTICA SOCIAL}

Como se ha mencionado anteriormente, Levy (1991) realizó un diagnóstico de la pobreza con datos de 1984, estableciendo una línea monetaria de pobreza extrema basada en un estándar de ingesta de calorías y sus cálculos mostraron que la incidencia de la pobreza extrema era de $19.5 \%$ a nivel nacional, $9.9 \%$ en las áreas urbanas y $37.2 \%$ en las áreas rurales. Aunque la canasta que se ha utilizado para medir la pobreza alimentaria en el periodo reciente no es estrictamente comparable con la utilizada por Levy, los datos que se presentan en la Gráfica 1 para 1992 y 1994 son consistentes con esas primeras mediciones de la pobreza alimentaria. En 1994, después de seis años de operación del programa SOLIDARIDAD, la pobreza persistía en niveles muy similares a los de 1984.

\section{Gráfica 1.}

México: incidencia de la pobreza alimentaria, 1992 a 2008

(Porcentaje de personas)

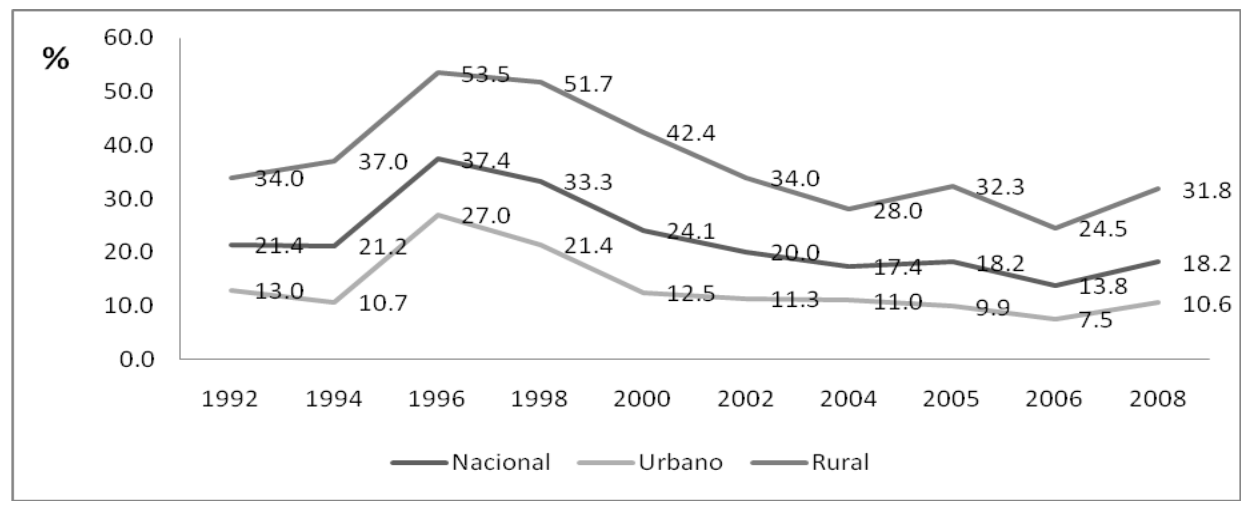

Fuente: CONEVAL.

Además, después de la crisis financiera y la macrodevaluación del peso mexicano de finales de 1994, Ernesto Zedillo inició su gobierno con un incremento significativo de la pobreza. En particular, la pobreza alimentaria afectaba en 1996 a 37 de cada 100 mexicanos a nivel nacional, a 27 de cada 100 mexicanos de las áreas urbanas y a un poco más de la mitad de la población en las áreas rurales. Al año siguiente, PROGRESA inicia sus operaciones focalizadas precisamente en los pobres alimentarios de las áreas rurales y al final del gobierno de Ernesto Zedillo, en el año 2000, la disminución de la pobreza había sido significativa, pero los niveles se mantenían todavía por encima de los observados a finales del gobierno de Carlos Salinas. En el año de 2000, después de cerca de 70 años en el poder, el PRI pierde la presidencia de la república y durante el gobierno de Vicente Fox, especialmente en el último año, se logran reducciones 
importantes en los niveles de incidencia de la pobreza alimentaria, ubicándose ya a niveles por debajo de los observados antes de la crisis de 1994. Sin embargo, en los dos primeros años del gobierno de Felipe Calderón ocurre un repunte de la pobreza, impulsado principalmente por el incremento en 2008 en los precios mundiales de los alimentos. De esta manera, las acciones y los programas de la política social en los últimos catorce años no han sido suficientes para eliminar el problema de la pobreza en México.

¿En dónde buscar las causas de esta persistencia de la pobreza a pesar de los esfuerzos del gobierno mexicano -desde PRONASOL hasta OPORTUNIDADES- para combatirla? Una parte importante de la respuesta a esta pregunta se encuentra en la propia racionalidad neogerencial, asistencialista y focalizada de la política social, pues estos programas y sus apoyos monetarios marginales están orientados a transformar las capacidades de los individuos sin crear distorsiones en el sistema de precios y para que los pobres con nuevas capacidades se incorporen a la vida productiva (Levy, 1991). La otra parte de la respuesta está en el desempeño de la economía (ver el Cuadro1) y en la actitud de la sociedad frente a la desigualdad social, que tiende a perpetuarla.

\section{Cuadro 1}

\begin{tabular}{|c|c|c|c|c|c|}
\hline \multicolumn{6}{|c|}{ México: comportamiento de los ingresos y los precios, 1990-2005 } \\
\hline Indicadores & $1990-1994$ & 1994-1995 & $1995-2000$ & $2000-2005$ & $1990-2005$ \\
\hline $\begin{array}{l}\text { Producto Interno Bruto } \\
\text { (PIB) })^{1}\end{array}$ & 3.7 & -6.2 & 6.1 & 1.9 & 3.6 \\
\hline PIB per cápita ${ }^{I}$ & 1.6 & -8.0 & 3.7 & 0.7 & 1.3 \\
\hline Inflación ${ }^{2}$ & 13.4 & 52.0 & 22.9 & 4.9 & 35.0 \\
\hline \multicolumn{6}{|l|}{ Salarios Reales } \\
\hline Mínimo $^{2}$ & -3.9 & -13.2 & -2.2 & 0.7 & -2.2 \\
\hline Contractual $^{3}$ & -0.7 & -16.0 & -4.8 & 2.8 & -1.4 \\
\hline Manufactureros $^{2}$ & 7.7 & -23.3 & 1.8 & 1.8 & 1.3 \\
\hline Ingresos laborales ${ }^{2}$ y & 1.4 & -13.1 & 8.0 & 1.1 & 0.5 \\
\hline \multicolumn{6}{|c|}{${ }^{1}$ Tasa media de crecimiento anual del promedio simple del PIB trimestral en el año, en pesos de 2002.} \\
\hline \multicolumn{6}{|c|}{${ }^{2}$ Tasa media de crecimiento anual del índice correspondiente. } \\
\hline \multicolumn{6}{|c|}{$\begin{array}{l}{ }^{3} \text { Promedio del incremento de los salarios contractuales reales ex-post (\%). } \\
{ }^{4} \text { Corresponden a la Encuesta Nacional de Ingreso y Gasto de los Hogares (ENIGH) de 1992-1994, 1994- } \\
1996,1996-2000,2000-2005 \text { y1992-2005 respectivamente. }\end{array}$} \\
\hline
\end{tabular}

Fuente: CONEVAL

En el Cuadro 1 se puede observar que durante el período posterior a la gran crisis de 1982 y a los derivados ajustes estructurales llevados a cabo por el gobierno, el desempeño de la economía en la generación de ingresos no ha sido suficiente para compensar los efectos del crecimiento de los precios. Tomando todo el período de 19902005, la economía creció a un promedio anual de apenas 3.6\% y el ingreso per cápita lo hizo a una tasa de $1.3 \%$, mientras que la inflación creció a una tasa media anual de $35 \%$; 
como resultado, ocurrieron contracciones anuales en los ingresos mínimos y contractuales y los ingresos laborales y manufactureros mostraron crecimientos muy modestos. En el mismo periodo, el índice de Gini, con el cual se mide la desigualdad en la distribución del ingreso, ha permanecido prácticamente constante en un valor alrededor de 0.53 .

Para terminar de tener una visión del tamaño de los desafíos persistentes de la política social en México, en el Cuadro 2 se presenta la información generada recientemente por el CONEVAL acerca de los rezagos en el disfrute de la población de los derechos sociales reconocidos en la Ley General de Desarrollo Social.

Cuadro 2.

México: indicadores de carencias sociales, 2008

\begin{tabular}{|lcc|}
\hline Indicadores de carencias sociales & Porcentaje & $\begin{array}{c}\text { Millones de } \\
\text { personas }\end{array}$ \\
\hline Rezago educativo & 21.7 & 23.2 \\
Acceso a los servicios de salud & 40.7 & 43.4 \\
Acceso a la seguridad social & 64.7 & 69.0 \\
Calidad y espacios de la vivienda & 17.5 & 18.6 \\
Acceso a los servicios básicos en la & & \\
vivienda & 18.9 & 20.1 \\
Acceso a la alimentación & 21.6 & 23.1 \\
\hline
\end{tabular}

Fuente: CONEVAL

La información contenida en este cuadro muestra también los efectos devastadores de una estructura económica que no ofrece oportunidades de desarrollo laboral y social, pues los rezagos sociales más importantes se encuentran precisamente en las áreas de la seguridad social y la salud -ambas muy ligadas al empleo de las personas-, en las que el $64.7 \%$ (equivalente a 69 millones de mexicanos) y el $40.7 \%$ (equivalente a 43.4 millones de mexicanos) de la población no cuenta respectivamente con algún esquema de protección en seguridad social y salud.

\section{CONSIDERACIONES FINALES}

El examen de los enunciados y los resultados de la política social en el periodo reciente muestra que la pobreza en México se ha convertido ya un viejo problema y que el gobierno no ha tenido la eficacia suficiente para erradicarlo. Aún en años en los que las condiciones macroeconómicas fueron relativamente favorables para el desarrollo social, la pobreza ha persistido, pues un porcentaje importante de la población mexicana en áreas rurales y urbanas enfrenta cotidianamente el problema de la carencia de recursos y la falta de oportunidades sociales para su desarrollo. Si a estos pobres les sumamos los pobres que se agregan ante contingencias económicas nacionales como las que han ocurrido en 1994 
y más recientemente en 2008 , la pobreza se amplía y llega a afectar a un sector muy amplio de la población.

La experiencia reciente muestra también las limitaciones de la política social focalizada y asistencialista que se orienta hacia el mejoramiento de las capacidades de los individuos que son pobres o excluidos socialmente. Aunque la focalización es una estrategia racional cuando los recursos públicos son escasos, los enfoques focalizados y asistencialistas alcanzan rápidamente sus límites si las capacidades de los sujetos no son convertidas en oportunidades de desarrollo individual y colectivo y si las estructuras sociales y normativas en las que se sustenta la desigualdad social no son modificadas.

El gobierno tiene la responsabilidad de combatir la pobreza con eficacia, pero ya no puede hacerlo solo. Para lograrlo, necesita de la participación social efectiva de los diferentes actores sociales, nacionales e internacionales, con capacidad de gestión y de poder para cambiar las cosas. Apenas en 2008, la primera crisis económica del siglo XXI nos ha vuelto a recordar la insuficiencia del gobierno para dirigir el proceso de desarrollo económico y abatir la pobreza en un nuevo y todavía más complejo contexto de producción e intercambio de bienes y servicios caracterizado por la globalización y por la creciente interdependencia entre los procesos, los agentes económicos, las regiones y los países.

El examen de la participación social en las instituciones de la política social nos permite sugerir que para darle mayor viabilidad institucional a la política social es necesario aumentar la densidad de la participación social y mejorar la calidad de dicha participación, de tal manera que el sistema en su conjunto funcione de manera más adecuada, creando condiciones favorables para que su cumplan los enunciados de la política social. En la nueva gobernanza se hace mucho énfasis en que los actores participantes de la misma deben poseer poder y capacidad de gestión, aunque las relaciones entre ellos no sean necesariamente simétricas y aunque los resultados de la deliberación conjunta no dejen enteramente satisfechos a todos los participantes en el proceso de gobernar, empezando por el propio gobierno.

Ante la debilidad estructural de las finanzas del gobierno, es necesario recuperar parte de la capacidad fiscal perdida por las sucesivas crisis económicas y dejar de depender de los recursos petroleros, los cuales siguen representando una porción importante de la recaudación total y usar esa nueva capacidad con eficacia en la solución de los problemas sociales y en la creación de oportunidades de desarrollo para la población.

En el período reciente, la fragilidad del crecimiento económico ha impedido que los ingresos de la población crezcan y contribuyan de manera suficiente a disminuir la pobreza absoluta y a mejorar la posición relativa de los pobres en la sociedad. Para aumentar el ingreso per cápita y los ingresos laborales de la población es también urgente recuperar y mantener el crecimiento económico y mejorar su calidad.

\section{BIBLIOGRAFÍA}

AGUILAR, L. F. (2006): Gobernanza y gestión pública, México, Fondo de Cultura Económica. 
- (2008a): "Marco para el análisis de las políticas públicas", Administración \& ciudadanía, vol. 3, n², pp. 9-28.

- (2008b): "Gobernanza: normalización conceptual y nuevas cuestiones", Cuadernos de Liderazgo, $\mathrm{n}^{\circ} 17$, Barcelona, ESADE.

- (2010): "El futuro de la gestión pública y la gobernanza después de la crisis", en Frontera Norte, vol. $22, n^{\circ} 43$, enero-junio, pp. 187-213.

BERGER, P. L. y LUCKMANN, Th. (1967): The social construction of reality, San Francisco, Anchor Books.

COADY, D. (2003): "Alleviating structural poverty in developing countries: the approach of PROGRESA in México", World Bank Working Paper Series.

CONSEJO CONSULTIVO DEL PROGRAMA NACIONAL DE SOLIDARIDAD (1994): El Programa Nacional de Solidaridad, México, Consejo Consultivo del Programa Nacional de Solidaridad.

COMISIÓN ECONÓMICA PARA AMÉRICA LATINA CEPAL (1977): Estudio económico para América Latina 1976, Santiago de Chile, Naciones Unidas.

- (1978): Estudio económico para América Latina 1977, Santiago de Chile, Naciones Unidas.

- (1984): Estudio económico para América Latina 1983, Santiago de Chile, Naciones Unidas.

- (1985): Estudio económico para América Latina 1983, Santiago de Chile, Naciones Unidas.

- (1988): Estudio económico para América Latina 1986, Santiago de Chile, Naciones Unidas.

- (1989): Estudio económico para América Latina 1988, Santiago de Chile, Naciones Unidas.

- (1990): Estudio económico de América Latina y el Caribe 1989, Santiago de Chile, Naciones Unidas.

CORTÉS, F.; HERNÁNDEZ LAOS, E. y RUBALCAVA, R.M. (1990): "Distribución de los ingresos salariales en el sector formal de la economía mexicana", México en el umbral del milenio, México, El Colegio de México, pp. 273-306.

DÍAZ CAYEROS, A. y MAGALONI, B. (2003): "The politics of public spending -Part II. The Programa Nacional de Solidaridad (PRONASOL) in Mexico", Background paper for the World Bank Development Report 2004.

DUNLEAVY, P. y HOOD, C. (1994): "From old public administration to new public management", Public money and management, julio-septiembre, pp. 9-16.

GIDDENS, A. (1984): The constitution of society, University of California Press, Berkeley.

HERNÁNDEZ LAOS, E. (1999): "Condicionantes macroeconómicos de la evolución de la pobreza en México", Boltvinik, J. y Hernández Laos, E. (coords.), Pobreza y distribución del ingreso en México, México, Siglo XXI Editores, pp. 119-153.

HOOD, C. (1991): “A public management for all seasons?” Public administration, $\mathrm{n}^{\circ}$ 69, pp. 3-19.

KOOIMAN, J. (2004): “Gobernar en gobernanza”, Instituciones y desarrollo, n 16, pp. 171-194.

KOOIMAN, J.; BAVINCK, M.; CHUENPAGDEE, R.; MAHON, R. y PULLIN, R. (2008): "Interactive governance and governability: an introduction", Journal of transdisciplinary environmental studies, vol. 7, $\mathrm{n}^{\circ}$ 1, pp. 2-11.

LEVY, S. (1991): "Poverty alleviation in Mexico", World Bank Working Paper Series.

MARINI, R. M. (1984): "La América Latina ante la crisis mundial", Lecturas 55. La crisis internacional y la América Latina", Méndez, S. (coord.), México, CIDE/El Trimestre Económico, pp. 35-52.

ORGANIZACIÓN PARA LA COOPERACIÓN Y EL DESARROLLO ECONÓMICOS (2009):

Estudio de la OCDE sobre el proceso presupuestario en México, México, OCDE.

OROZCO, M. y HUBERT, C. (2005): "La focalización en el programa de desarrollo humano Oportunidades de México", World Bank Working Paper Series.

OSBOURNE, D. y GAEBLER, T. (1992): Reinventing government. How the entrepreneurial spirit is transforming the public sector, New York, Penguin Books.

OSBOURNE, D. y PLASTRIK, P. (1999): Banishing bureaucracy. The five strategies for reinventing government, San Francisco, Jossey Bass. 
PZEWORSKI, A. (1982): "La teoría sociológica y el estudio de la población: reflexiones sobre los trabajos de la Comisión de Población y Desarrollo de CLACSO", Reflexiones teóricometodológicas sobre investigaciones en población, México, El Colegio de México, pp. 59-99.

REYGADAS, L. (2008): La apropiación. Destejiendo las redes de la desigualdad, México, Anthropos/Universidad Autónoma Metropolitana.

WEBER, M. (1996): Economía y sociedad, México, Fondo de Cultura Económica.

\section{Breve currículo:}

\section{Félix Acosta Díaz}

Doctor en Ciencias Sociales con Especialidad en Estudios de Población por El Colegio de México. Investigador del Departamento de Estudios de Población de El Colegio de la Frontera Norte (El COLEF) desde 1989, donde imparte clases de estadística aplicada a las ciencias sociales, metodología de la investigación, gestión pública y evaluación de políticas públicas. Sus temas de investigación son la familia, la pobreza y las políticas públicas. En 2006 fue electo como uno de los seis Investigadores Académicos del Consejo Nacional de Evaluación de la Política de Desarrollo Social (CONEVAL) durante el periodo 2006-2010. Sus responsabilidades más importantes en el CONEVAL fueron: normar y evaluar la política de desarrollo social de la administración pública federal y desarrollar e implementar una metodología oficial para la medición multidimensional de la pobreza en México. 